\title{
Erdöl und Bergbau: Mobilisierung der afrikanischen Zivilgesellschaft
}

\section{Catherine Morand}

\section{(2) OpenEdition}

\section{Journals}

Electronic version

URL: http://journals.openedition.org/sjep/376

DOI: $10.4000 /$ sjep.376

ISSN: 1663-9677

\section{Publisher}

Institut de hautes études internationales et du développement

\section{Printed version}

Date of publication: 1 novembre 2007

Number of pages: 103-105

ISBN: 978-2-88247-069-0

ISSN: $1660-5926$

\section{Electronic reference}

Catherine Morand, «Erdöl und Bergbau: Mobilisierung der afrikanischen Zivilgesellschaft »,

Schweizerisches Jahrbuch für Entwicklungspolitik [Online], 26-2 | 2007, Online erschienen am: 14 Juni 2010, abgerufen am 07 September 2020. URL : http://journals.openedition.org/sjep/376 ; DOI :

https://doi.org/10.4000/sjep.376 


\title{
Erdöl und Bergbau: Mobilisierung der afrikanischen Zivilgesellschaft
}

\author{
Catherine Morand*
}

n immer zahlreicheren afrikanischen Ländern, die über bedeutende Erdölvorkommen und sonstige Bodenschätze verfügen, ist eine Mobilisierung der Zivilgesellschaft im Gange, die mit vorbildlichem Mut von den eigenen Regierungen wie auch von den Fördergesellschaften mehr und echte Transparenz fordert, um eine Verbesserung der Lebensumstände der betroffenen Bevölkerung zu ermöglichen. Das konnte die Verfasserin im März 2007 anlässlich eines Treffens von Vertreterinnen und Vertretern aller Erdöl- und Bergbauländer Afrikas feststellen.

Am 26. April 2007 kündigte Michel Yoboué, Leiter für die Elfenbeinküste der Kampagne „Publish What You Pay" (Veröffentliche, was du zahlst), an einer Pressekonferenz in Abidjan den Start einer nationalen Sensibilisierungskampagne an, um „Rechenschaft über die Verwendung der Ölgelder zu verlangen“. Er wies dabei auf die herrschende Intransparenz in Zusammenhang mit der Verwendung des Erdölsegens in der Elfenbeinküste hin, „in einem Land, dass trotz einer Produktion von 90000 Barrel pro Tag noch immer nicht zu den Erdöl produzierenden Ländern gezählt wird", während beispielsweise Kamerun mit einer halb so starken Förderung auf der entsprechenden IWF-Liste stehe. „Wir möchten den Forderungen der Zivilgesellschaft nach Transparenz im Umgang mit dem Ölsektor Gehör verschaffen", meinte Michel Yoboué weiter.

\section{Wahrlich revolutionär}

In dem undurchsichtigen Umfeld der ivorianischen und allgemein afrikanischen Erdöl- und Bergbauindustrie ist das wahrlich eine Revolution. Besonders zu begrüssen ist die zunehmende Zahl von Initiativen zur Förderung neuer Spielregeln, welche die Bevölkerung der Erdölund Bergbaustaaten in den Genuss einer transparenten Verwaltung der Erlöse bringen und so den Direktbetroffenen verbesserte Lebensbedingungen verschaffen sollen. Denn in diesen Wirt- schaftssektoren erfährt Afrika südlich der Sahara zurzeit eine Investitionswelle, wie sie der Kontinent bisher nie gekannt hat, und der Geldsegen, der sich daraus ergibt, übersteigt bei weitem den Mittelzufluss in Form von Entwicklungs- und Finanzhilfe. Ziel der Initiativen ist es, den „Fluch des schwarzen Goldes" zu brechen, das den afrikanischen Erdöl- und Bergbauländern bisher hauptsächlich Umweltkatastrophen und Korruption gebracht und zur Festigung der Macht autoritärer Regimes beigetragen hat.

Michel Yoboué gehörte zu den zahlreichen Vertreterinnen und Vertretern von Organisationen der afrikanischen Zivilgesellschaft an einem Treffen der Mitgliedsorganisationen der internationalen Kampagne "Publish What You Pay" (PWYP) im März 2007 in Limbe, Kamerun. Ziel dieser Zusammenkunft waren die Erarbeitung einer gemeinsamen Strategie und die Aneignung der für den Kampf erforderlichen Fachkenntnisse.

Ich nahm als Vertreterin von Swissaid, Schweizerische Stiftung für Entwicklungszusammenarbeit, an diesem Treffen teil. Swissaid ist in diesem Themenbereich über ihre Partnerorganisationen im Tschad, im Niger und in Guinea-Bissau stark engagiert. Angefangen hatte dieses Engagement mit der Unterstützung tschadischer Vereinigungen bei ihrem Kampf um eine Verwendung der

* Journalistin, Leiterin der Antenne romande von Swissaid, Lausanne.

Dieser Bericht zeugt von der Mobilisierung der afrikanischen Bevölkerung im Rahmen der Initiative für Transparenz in der Rohstoffwirtschaft (EITI), der in diesem Dossier auch der Beitrag von Gilles Carbonnier gewidmet ist. 
Ölerträge im Sinne einer tatsächlichen Verbesserung der Lebensbedingung der Bevölkerung. Swissaid unterstützt somit seit der Jahrhundertwende die Bemühungen der tschadischen Zivilgesellschaft, sich zu Wort zu melden. Dasselbe gilt inzwischen für den Niger und Guinea-Bissau, zwei Länder, in denen Ölbohrungen an der Tagesordnung sind. Swissaid war immer bestrebt, alle von der Frage einer transparenten Verwaltung der Erdölerträge betroffenen Parteien zusammenzuführen, sei dies im Rahmen einer Reise von Schweizer Parlamentsmitgliedern, Journalistinnen und Journalisten in den Tschad im April 2004 oder anlässlich verschiedener Konferenzen in der Schweiz, im Niger und in Guinea-Bissau. So auch in Genf, wo sich im November 2006 auf Einladung von Swissaid Vertreterinnen und Vertreter von Erdölgesellschaften, internationalen Organisationen, Erdölstaaten, der Zivilgesellschaft und mehreren NRO trafen, um sich mit der Tatsache auseinanderzusetzen, dass Erdöl für Afrika nicht zwangsweise ein Schicksalsschlag sein muss.

Die afrikanischen Länder spielen im Übrigen eine gewichtige Rolle bei der PWYP-Kampagne, der sich zahlreiche Organisationen aus dem Umwelt-, Menschenrechts- und Gewerkschaftsbereich angeschlossen haben. Ihnen allen ist ein Anliegen gemeinsam: der Kampf um eine transparente Verwaltung der Gelder aus der Öl- und Bergbauindustrie. „Zu wissen, wie viel Geld die Regierungen einnehmen und wie viel die Gesellschaften zahlen, ist eine wichtige Voraussetzung, um die für die Verwaltung dieser Gelder zuständigen Behörden zur Rechenschaft zu ziehen", meint Matteo Pellegrini, Afrikakoordinator von PWYP, der Organisatorin dieser Fortbildungsveranstaltung. Die 2002 gestartete Kampagne wird heute von über 300 NRO weltweit unterstützt.

\section{Eine Gegenbewegung entsteht}

Diese Bündnisse machen heute Druck auf ihre jeweiligen Regierungen, um sie zum Beitritt zur Extractive Industries Transparency Initiative (Initiative für Transparenz in der Rohstoffwirtschaft, EITI) zu bewegen, welche dieselben Ziele verfolgt. Diese Initiative - sie wurde 2002 anlässlich des Weltgipfels für nachhaltige Entwicklung in Johannesburg von Tony Blair ins Leben gerufen - setzt auf eine Absprache zwischen Regierungen, Erdölfirmen, internationalen Organisationen und der Zivilgesellschaft, um die Einnahmen aus den Erdöl-, Erdgas- und Bergbauunternehmen transparenter zu gestalten. Dahinter stecken die Überzeugung, dass ein solches Vorgehen eigentlich im Interesse aller Beteiligten liegt, und das Bestreben, „nigerianische Verhältnisse“ mit Entführungen, Sabotageakten und einem praktisch allgegenwärtigen Elend zu vermeiden.

Besonders zahlreich waren in Limbe die Organisationen aus dem Tschad vertreten. Die Mobilisierung der tschadischen Zivilgesellschaft nahm ihren Anfang, als die Ausbeutung der Erdölvorkommen des Landes in Erwägung gezogen wurde, und hat stark zum Aufkommen einer Gegenbewegung beigetragen; sie gilt bis zum heutigen Tage als Vorbild. Die Weltbank hatte zunächst zu dieser Entwicklung beigetragen und sich mit ihrem ganzen Einfluss für die gesetzliche Verankerung einer transparenten Verwaltung der Erdöleinnahmen eingesetzt. Später wurde dieses weltweit einzigartige Gesetz, das Gesetz 001, allerdings ausgehöhlt und durch das Gesetz 002 ersetzt, mit dem insbesondere der Fonds für zukünftige Generationen aufgelöst wurde. Die Weltbank, die nicht in der Lage war, die Einhaltung der vertraglichen Verpflichtungen bei den tschadischen Behörden durchzusetzen, hat in den Augen der Vertreterinnen und Vertreter der Zivilbevölkerung jede Glaubwürdigkeit verloren: Sie verfügt inzwischen über keine Druckmittel mehr, um von der tschadischen Regierung eine transparente Verwaltung der Ölgelder einzufordern. „Wir setzen nun unsere Hoffnung auf den Druck der internationalen Kampagne PWYP, um die Behörden im Tschad dazu zu bewegen, sich der EITI anzuschliessen", erklärt Gilbert Maoundonodji, Leiter von GRAMP/TC, einer von Swissaid unterstützten tschadischen NRO, die sich an dieser Kampagne beteiligt - wie übrigens die meisten Organisationen der tschadischen Zivilgesellschaft, die sich für eine transparente Verwaltung der Ölgelder einsetzen.

Von der tschadischen Zivilgesellschaft lernen

Dank ihrer frühen Mobilisierung auf diesem Gebiet erlangte die tschadische Zivilgesell- 
schaft einen Wissensvorsprung. Ihre Erfahrungen sind inzwischen auch im übrigen Afrika gefragt. So hat sich Gilbert Maoundonodji, heute Afrikavertreter beim Internationalen EITI-Rat mit Sitz in Oslo, persönlich an der Lancierung der Initiative in der Republik Kongo (Kongo Brazzaville) und Mauretanien beteiligt. „Das Fachwissen der tschadischen Zivilgesellschaft ist heute eine wesentliche Antriebskraft für die PWYP-Kampagne in ganz Afrika", betont er. Die Fortbildungsveranstaltung in Limbe hatte unter anderem das Ziel, die Sachkompetenz der Vertreterinnen und Vertreter der Zivilgesellschaft zu verbessern und sie somit in die Lage zu versetzen, ihre Rolle in ihrer ganzen Tragweite wahrzunehmen. Dazu ist es beispielsweise erforderlich, zu wissen, wie man das Budget eines Staates und die Verträge mit den Erdöl- und Bergbaugesellschaften liest, versteht und entziffert, über alle Aspekte der EITI-Initiative gut unterrichtet zu sein, die Rechtslage in den jeweiligen Ländern zu kennen usw. „Längerfristig ist die Neuverhandlung einiger Verträge erforderlich, denn die Gebühren, welche die Fördergesellschaften unseren Staaten ausrichten, sind oft lachhaft", beklagt Steve Emmanuel Manteaw von der ghanaischen Vereinigung Integrated Social Development Centre, die sich mit den Auswirkungen des Rohstoffabbaus auf Umwelt und Gesellschaft in Ghana befasst.

Auch die mutige Mobilisierungsarbeit unter schwierigsten Bedingungen, die von Organisationen der Zivilgesellschaft in der Demokratischen Republik Kongo geleistet wird, hat durch die Extractive Industries Transparency Initiative eine Verstärkung erfahren. „Unser Engagement in Fragen der Rohstoffgewinnung geht auf das Jahr 2000 zurück", erzählt Jean-Pierre Muteba, Leiter einer gewerkschaftlichen Organisation in Lubumbashi. „Die EITI ist da gerade zum richtigen Zeitpunkt gekommen. Sie gibt uns die Möglichkeit, ein Bergbaugesetz anzuprangern, das in einer Zeit des politischen Umbruchs auf Druck der Weltbank hastig verabschiedet wurde und die Interessen unseres Landes in keiner Weise berücksichtigt." Um den Ausverkauf der Bodenschätze Kongos zu verhindern und bessere Arbeitsbedingungen zu erkämpfen, hat er im Bergbausektor der rei- chen Provinz Katanga mehrere Streikbewegungen initiiert. Damit begehrte er gegen die korrupten Lokalbehörden wie auch gegen die Beutezüge unzähliger Bergbaugesellschaften auf, welche die nahezu völlige Abwesenheit jeglicher staatlichen Kontrolle ausnutzen, um sich über den Kupfer-, Diamant-, Coltan- und Kobaltreichtum dieser Region herzumachen. Da Jean-Pierre Muteba immer wieder Opfer militärischer Verfolgung wurde, zahlt inzwischen eine holländische NRO bewaffnete Wachen, die vor seinem Haus stehen und so inm und seiner Familie ein Minimum an Sicherheit bieten. „Wir müssen für uns und unsere Kinder kämpfen, um die Plünderung unserer Bodenschätze zu verhindern; denn wir können nirgendwo anders hin", erklärt er weiter.

\section{Verfolgung, Druck, Verhaftung ...}

Wer sich in Afrika im hochsensiblen Sektor der Rohstoffgewinnung für mehr Transparenz bei der Verwaltung der Einnahmen einsetzt, sieht sich in der Tat regelmässigen Druckversuchen, Drohungen oder auch Verhaftungen ausgesetzt. Ausgeübt wird dieser Druck in erster Linie von den Machthabern des Landes, zum Teil aber auch von Unternehmen. „Nur weil wir verfolgt werden, werden wir den Kampf noch lange nicht aufgeben", meint jedoch Samuel Nsikabaka, Universitätsprofessor in Brazzaville. Sein Land kann sinnbildlich für das gesamte Drama stehen, das mit dem Begriff "Fluch des Erdöls" bezeichnet wird: das paradoxe Phänomen, dass je reicher ein Land an natürlichen Ressourcen, desto tiefer das beschämende Elend seiner Bevölkerung ist. Samuel Nsikabaka hat anlässlich der Parlamentswahlen in Kongo im Juni 2007 von den Kandidierenden Rechenschaft über die Verwaltung der Erdölerträge verlangt. „Der Staat soll uns sagen, wie diese Gelder ausgegeben wurden", fordert er. Seine Familie hat er nach Frankreich in Sicherheit gebracht, denn schon zweimal ist sein Haus von „Unbekannten" in Brand gesteckt worden. Zwei Mitglieder der PWYP-Koalition der Republik Kongo, Christian Mounzéo und Brice Mackosso, stehen nach ihrer Festnahme, willkürlichen Inhaftierung und Verurteilung wegen Verleumdung des Staatsoberhauptes weiterhin unter Hausarrest. 